\title{
PARAMETRIC RESONANCE IN SYSTEMS WITH SMALL DISSIPATION†
}

\author{
A. A. MAILYBAYEV and A. P. SEYRANIAN \\ Moscow
}

(Received 14 December 2000)

\begin{abstract}
A linear oscillatory system having multiple degrees of freedom with periodic coefficients is considered. The system involves three independent parameters: the frequency and amplitude of the periodic exitation and a parameter of the dissipative forces, the last two being assumed small. Instability of the trivial solution (parametric resonance) is investigated. For an arbitrary periodic exitation matrix and a positive-definite matrix of the dissipative forces, general expressions are obtained for the domains of fundamental and combination resonances. Two special cases of the periodic exitation matrix, frequently encountered in applications, are studied: a symmetric matrix, and a time-independent matrix multiplied by a scalar periodic function. It is proved that in the first case the system is subject only to fundamental and sum-type combination resonances; in the second case fundamental resonance and sum or difference type combination resonance may occur, depending on the sign of a certain constant. It is shown that in both cases the resonance domains in the first approximation are cones in three-dimensional parameter space. Examples considered are the problem of the dynamic stability of a two-dimensional bending mode of an elastic beam subject to periodic torques, and the problem of the stability of an elastic rod of variable cross-section compressed by a periodic longitudinal force. (ㅇ) 2002 Elsevier Science Ltd. All rights reserved.
\end{abstract}

The phenomenon of parametric resonance is observed in many physical systems. One of the essential factors that determine the onset of resonance is energy dissipation. In this paper it is assumed that the dissipative forces and the amplitude of the periodic exitation are small. The formulae derived for the combination resonance domains (cones in parameter space) contain two frequencies and two corresponding oscillatory modes of the unperturbed conservative system, corresponding to a resonance excitation frequency. In the fundamental resonance, only one frequency and mode of uscillation are needed. This enables us to treat parametric resonance as the mutual interaction of the oscillation frequencies (modes) of a conservative system subject to periodic exitation at a definite frequency.

The relations obtained for the parametric resonance domains enable us to analyse the effect of increasing the frequencies of natural oscillations and resonance number on the instability domain. In particular, it is shown that as the resonance number increases the instability cone becomes narrower, while the cone axis is straightened. In the majority of publications on parametric resonance it is assumed that the value of the periodic matrix, averaged over a period, is zero. In this paper, this restriction is dropped. The existence of a non-zero average has a substantial effect on the resonance domain, making the axes of the instability cones deviate from the vertical.

In the case of non-resonance excitation frequencies, small dissipative forces stabilize the system, i.e., they make it asymptotically stable. For given dissipative forces, the boundary of the parametric resonance domain in the first approximation is described by a hyperbola; we will find the minimum (critical) values of the amplitude of the periodic input at which resonance occurs, as well as the corresponding excitation frequencies. Formulae for resonance zones when the dissipation parameter tends to zero will also be derived, and the paradox of the destabilization of the system by infinitesimal dissipative forces will be discussed.

Of previous studies, we should mention first of all the analysis of nearly Hamiltonian systems [1], as well as systems reduced to normal coordinates of a conservative system (which require a knowledge of the transition matrix) [2-5]. The method for investigating parametric resonance domains proposed in this paper is based on an analysis of the behaviour of multipliers and an evaluation of the derivatives of the monodromy matrix with respect to the parameters $[6,7]$. A system with one degree of freedom and three independent parameters was considered in [8]. 


\section{FORMULATION OF THE PROBLEM}

Consider a linear oscillatory system with periodic coefficients

$$
\mathbf{M} \ddot{\mathbf{y}}+\gamma \mathbf{D} \dot{\mathbf{y}}+(\mathbf{C}+\delta \mathbf{B}(\Omega t)) \mathbf{y}=\mathbf{0}
$$

where $\mathbf{M}, \mathbf{D}$ and $\mathbf{C}$ are symmetric positive-definite $m \times m$ matrices of the masses, and the damping and potential forces, $\mathbf{B}(\tau)$ is the piecewise-continuous $2 \pi$-periodic matrix of parametric excitation; $\mathbf{y}=\left(y_{1}, \ldots, y_{m}\right)^{T}$ is the vector of generalized coordinates; and the dots denote differentiation with respect to time $t$.

We will investigate the stability of the trivial solution $y \equiv 0$ of system (1.1) as a function of the vector of three parameters $\mathbf{p}=(\gamma, \delta, \Omega)$, which describes the magnitude of the dissipative forces, the amplitude and frequency of periodic excitation, assuming that $\gamma$ and $|\delta|$ are both small. This assumption corresponds to the stipulation that system (1.1) is close to an autonomous conservative system. The parameters $\gamma$ and $\Omega$ are subject to the natural restrictions $\gamma \geqslant 0$ and $\Omega>0$.

We will write (1.1) as a system of first-order equations

$$
\begin{aligned}
& \dot{\mathbf{x}}=\mathbf{A}(\Omega t) \mathbf{x} \\
& \mathbf{x}=\left\|\begin{array}{l}
\mathbf{y} \\
\dot{\mathbf{y}} \|
\end{array} \quad \mathbf{A}(\Omega t)=\right\| \begin{array}{cc}
0 & \mathbf{I} \\
-\mathbf{M}^{-1}(\mathbf{C}+\delta \mathbf{B}(\Omega t)) & -\gamma \mathbf{M}^{-1} \mathbf{D}
\end{array} \|
\end{aligned}
$$

The matrix $A(\Omega t)$, of order $2 m \times 2 m$, is a real periodic function with period $T=2 \pi / \Omega$. The matriciant of system (1.2) is defined as a matrix $\mathbf{X}(t)$ of order $2 m \times 2 m$ satisfying the following equation and initial conditions

$$
\dot{\mathbf{X}}=\mathbf{A}(\Omega t) \mathbf{X}, \quad \mathbf{X}(0)=\mathbf{I}
$$

where $\mathbf{I}$ is the identity matrix. The value of the matriciant at $t=\mathbf{T}$ is called the monodromy matrix F [1]:

$$
\mathbf{F}=\mathbf{X}(T)
$$

By the theorem on the dependence of the solution of a differential equation on the parameters, the monodromy matrix is a smooth function of the parameter vector $\mathbf{p}$. The eigenvalues (multipliers) $\rho$ and eigenvectors $\mathbf{w}$ of the monodromy matrix are determined from the equation

$$
\mathbf{F w}=\rho \mathbf{w}
$$

System (1.2) is asymptotically stable if all the multipliers lie inside the unit circle $|\rho|<1$ in the complex plane. If at least one multiplier lies outside the unit circle, the system is unstable [1].

If $\gamma=\delta=0$, system (1.1) is conservative:

$$
\mathbf{M} \ddot{\mathbf{y}}+\mathbf{C y}=\mathbf{0}
$$

Seeking a solution of Eq. (1.6) in the form $\mathbf{y}=\mathbf{u} \exp (i \omega t)$, we arrive at the eigenvalue problem

$$
\mathbf{C u}=\omega^{2} \mathbf{M u}, \quad \mathbf{u}^{T} \mathbf{M u}=1
$$

where the second equality is the normalization condition. From these equations we determine real values: the natural frequencics $\omega$ and modes of oscillation $u$. Let us assume that all the frequencies $\left.0,<\omega_{1}<\omega_{2}<\ldots<\omega_{m}\right)$ are different. Denote the corresponding eigenvectors by $\mathbf{u}_{j}(j=1, \ldots, m)$.

Matriciant (1.3) and monodromy matrix (1.4) have the following form when $\gamma=\delta=0[1]$

$$
\mathbf{X}(t)=\exp \left(\mathbf{A}_{0} t\right), \quad \mathbf{F}_{0}=\exp \left(\mathbf{A}_{0} T\right), \quad \mathbf{A}_{0}=\left\|\begin{array}{cc}
\mathbf{0} & \mathbf{I} \\
-\mathbf{M}^{-1} \mathbf{C} & \mathbf{0}
\end{array}\right\|
$$

The eigenvalues of the matrix $\mathbf{A}_{0}$, as well as the corresponding right and left eigenvectors $\mathbf{w}$ and $\mathbf{v}$, are defined by the relations 


$$
\mathbf{A}_{0} \mathbf{w}=\lambda \mathbf{w}, \quad \mathbf{v}^{T} \mathbf{A}_{0}=\lambda \mathbf{v}^{T}, \quad \mathbf{v}^{T} \mathbf{w}=\mathbf{l}
$$

The last equality is the normalization condition. It follows from (1.7)-(1.9) that the eigenvalues of the matrix $\mathbf{A}_{0}$ are $\lambda_{j}, \lambda_{j}= \pm i \omega_{j}(j, \ldots, m)$. The right and left eigenvectors for $\lambda_{j}=i \omega_{j}$ are

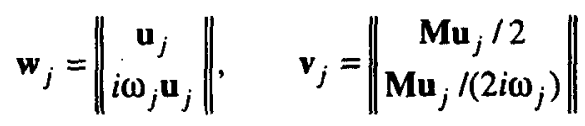

It is well known from matrix theory [1] that the multipliers of the matrix $\mathbf{F}_{0}=\exp \left(\mathbf{A}_{0} T\right)$ are

$$
\rho_{j}, \bar{\rho}_{j}=\exp \left( \pm i \omega_{j} T\right)=\exp \left( \pm i 2 \pi \omega_{j} / \Omega\right), \quad j=1, \ldots, m
$$

and moreover the eigenvectors of the matrices $\mathbf{F}_{0}$ and $\mathbf{A}_{0}$ are identical. Thus, the vectors $\mathbf{w}_{j}$ and $\mathbf{v}_{j}$ of (1.10) are the right and left eigenvectors corresponding to the multiplier $\rho_{j}$ of the monodromy matrix $\mathbf{F}_{0}$. They satisfy the relations

$$
\mathbf{F}_{0} \mathbf{w}_{j}=\rho_{j} \mathbf{w}_{j}, \quad \mathbf{v}_{j}^{T} \mathbf{F}_{0}=\rho_{j} \mathbf{v}_{j}^{T}, \quad \mathbf{v}_{j}^{T} \mathbf{w}_{j}=1
$$

The complex-conjugate multipliers $\bar{\rho}_{j}=\exp \left(-i \omega_{j} T\right)$ correspond to the complex-conjugate eigenvectors $\overline{\mathbf{w}}_{j}$ and $\overline{\mathbf{v}}_{j}$.

Since all the multipliers $\rho_{j}$ of (1.11) lie on the unit circle $|\rho|=1$, the stability of system (1.1) for non-zero $\gamma$ and $\delta$ is determined by perturbations of all the multipliers $\rho_{j}$. In the case of the general position, all the $\rho_{j}$ are different. Multiple multipliers arise for critical values of the frequency, which are

$$
\begin{gathered}
\Omega=2 \omega_{j} / k, \quad j=1, \ldots, m ; \quad k=1,2, \ldots \\
\Omega=\left(\omega_{j} \pm \omega_{l}\right) / k, \quad i, j=1, \ldots, m ; \quad j>l ; \quad k=1,2, \ldots
\end{gathered}
$$

Case (1.13) and (1.14) are called respectively a fundamental (simple) resonance (FR) and a combination resonance (CR) [1] and correspond to the existence of double multipliers $\rho=(-1)^{k}$ and $\rho=\exp \left(i \omega_{j} T\right)$, respectively. These multipliers are semi-simple, since they have two linearly independent eigenvectors $\mathbf{w}_{j}$ and $\overline{\mathbf{w}}_{j}$ in case (1.13) and $\mathbf{w}_{j}$ and $\overline{\mathbf{w}}_{l}$ (or $\mathbf{w}_{j}$ and $\mathbf{w}_{l}$ for the negative sign) in case (1.14). If the frequency $\Omega$ is close to the critical value, the system may become unstable. The critical cases are therefore of particular interest. Multipliers of higher multiplicity arise only when there are rational relations among the quantities $\omega_{j} \pm \omega_{l}(j, l=1, \ldots, m ; j \geqslant l)$, where $j \neq l$ in the case $\omega_{j}-\omega_{l}$. These cases are not typical and will not be considered further, though they may be investigated in a similar way.

\section{THE BEHAVIOUR OF SIMPLE MULTIPLIERS}

Let us consider a simple multiplier $\rho_{j}=\exp \left(i \omega_{j} T_{0}\right)\left(T_{0}=2 \pi / \Omega_{0}\right)$ corresponding to the monodromy matrix $F_{0}$, for $\gamma=\delta=0$ and some value $\Omega=\Omega_{0}$. Investigation of the multiplier $\bar{\rho}_{j}=\exp \left(-p \omega_{i} T_{0}\right)$ is similar. As we know, a simple multiplier of a smooth family of matrices $\mathbf{F}(\mathbf{p})$ is a smooth function of the parameter vector $\mathbf{p}$. The derivative of the multiplier $\rho_{j}$ with respect to the parameter $p_{k}$ has the form [9]

$$
\frac{\partial p_{j}}{\partial p_{k}}=\mathbf{v}_{j}^{T} \frac{\partial \mathbf{F}}{\partial p_{k}} \mathbf{w}_{j}
$$

where the first derivations of the monodromy matrix are defined by the following expression $[6,7]$

$$
\frac{\partial \mathbf{F}}{\partial p_{k}}=\mathbf{F} \int_{0}^{T_{0}} \mathbf{X}^{-1} \frac{\partial \mathbf{A}}{\partial p_{k}} \mathbf{X} d t+\mathbf{A}\left(T_{0}\right) \mathbf{F} \frac{\partial T}{\partial p_{k}}
$$

Using formulae(1.8), (1.9), (1.12) and (2.2), we write (2.1) as 


$$
\frac{\partial \rho_{j}}{\partial p_{k}}=\rho_{j}\left(\int_{0}^{T_{0}} \mathbf{v}_{j}^{r} \frac{\partial \mathbf{A}}{\partial p_{k}} \mathbf{w}_{j} d t+i \omega_{j} \frac{\partial T}{\partial p_{k}}\right)
$$

where we have used the following property: the matrix $X=\exp \left(\mathbf{A}_{0}\right)$ has an eigenvalue $\exp (i \omega j)$ to which the right and left eigenvectors $\mathbf{w}_{j}$ and $\mathbf{v}_{j}$ correspond. Substituting the explicit form of the matrix $\mathbf{A}=\mathbf{A}(\Omega t)$ from (1.2) and the vectors $\mathbf{w}_{j}$ and $\mathbf{v}_{j}$ from (1.10) into Eq. (2.3), we find the derivatives of the multiplier with respect to the components of the parameter vector $\mathbf{p}=(\gamma, \delta, \Omega)$ at the point $\mathbf{p}_{0}=\left(0,0, \Omega_{0}\right)$

$$
\begin{aligned}
\frac{\partial \rho_{j}}{\partial \gamma} & =-\frac{\rho_{j} \pi \mathbf{u}_{j}^{T} \mathbf{D} \mathbf{u}_{j}}{\Omega_{0}}, \quad \frac{\partial \rho_{j}}{\partial \delta}=\frac{i \rho_{j} \pi c_{0}^{(j j)}}{\omega_{j} \Omega_{0}}, \quad \frac{\partial \rho_{j}}{\partial \Omega}=-\frac{2 i \rho_{j} \pi \omega_{j}}{\Omega_{0}^{2}} \\
c_{0}^{(j j)} & =\frac{1}{2 \pi} \int_{0}^{2 \pi} \mathbf{u}_{j}^{T} \mathbf{B}(\tau) \mathbf{u}_{j} d \tau
\end{aligned}
$$

Then in the neighbourhood of the point $\mathbf{p}_{0}$ the multiplier $\rho_{j}(\mathbf{p})$ can be represented in the form

$$
\begin{aligned}
& \rho_{j}(\mathbf{p})=\rho_{j}+\frac{\partial \rho_{j}}{\partial \gamma} \gamma+\frac{\partial \rho_{j}}{\partial \delta} \delta+\frac{\partial \rho_{j}}{\partial \Omega}\left(\Omega-\Omega_{0}\right)+o\left(\left\|\mathbf{p}-\mathbf{p}_{0}\right\|\right)= \\
& =\rho_{j}\left(1-\frac{\pi \mathbf{u}_{j}^{T} \mathbf{D} \mathbf{u}_{j}}{\Omega_{0}} \gamma+\frac{\pi c_{0}^{(j j)}}{\omega_{j} \Omega_{0}} \delta i-\frac{2 \pi \omega_{j}}{\Omega_{0}^{2}}\left(\Omega-\Omega_{0}\right) i\right)+o\left(\left\|\mathbf{p}-\mathbf{p}_{0}\right\|\right)
\end{aligned}
$$

These relations imply the following expression for the magnitude of the multiplier in the first approximation

$$
\left|\rho_{j}(\mathbf{p})\right|=1-\frac{\pi \mathbf{u}_{j}^{T} \mathbf{D} \mathbf{u}_{j}}{\Omega_{0}} \gamma+o\left(\left\|\mathbf{p}-\mathbf{p}_{0}\right\|\right)
$$

It follows from the assumption that the matrix $\mathbf{D}$ of dissipative forces is positive definite that the coefficient of $\gamma$ in (2.6) is negative. Consequently, the stability condition $|\rho|<1$ in the first approximation is the inequality

$$
\gamma>0
$$

Let us assume that $\gamma=0$ and consider the case when $\mathbf{B}(\tau)=\mathbf{B}^{T}(\tau)$ or $\mathbf{B}\left(\tau_{0}+\tau\right)=\mathbf{B}\left(\tau_{0}-\tau\right)$, where $\tau_{0}$ is a certain number. Then system (1.1) with $\gamma=0$ is Hamiltonian or reversible, respectively. In that case the characteristic polynomial of the monodromy matrix is reciprocal [1]; that is, if $\rho$ is a multiplier, then $1 / \rho$ is also a multiplier. Consequently, for $\gamma=0$ and small $|\delta|$ and $\left|\Omega-\Omega_{0}\right|$, the simple multipliers remain on the unit circle. Hence the plane $\gamma=0$ is the boundary of the asymptotic stability domain in the neighbourhood of the point $\boldsymbol{p}_{0}$. Thus, the introduction of small dissipative forces cases all the simple multipliers to move into the unit circle for small $|\delta|$ and $\left|\Omega-\Omega_{0}\right|$. This means that small dissipative forces stabilize system (1.1) with a small amplitude of parametric excitation for non-critical values of the frequency $\Omega$.

\section{PARAMETRIC RESONANCE DOMAIN}

Instability may arise at frequencies $\Omega$ close to the critical values (1.13) and (1.14). Under these conditions, double multipliers appear on the unit circle. Suppose the parametric excitation frequency $\Omega=\Omega_{0}$ satisfies the relation

$$
\omega_{j}+\omega_{l}=k \Omega_{0}
$$

at certain frequencies $\omega_{j}$ and $\omega_{l}$ of the conservative system (1.6) and for some natural number $k$. Note that this condition includes the case of fundamental resonance (1.13) when $j=l$ and the case of sum 
combination resonance (1.14) when $j>l$. The critical frequencies $\Omega_{0}=\left(\omega_{j}-\omega_{l}\right) / k$ corresponding to difference combination resonance will be considered below by analogy.

Condition (3.1) means that the two multipliers are identical:

$$
\rho_{j}=\bar{\rho}_{l}=\exp \left(i \omega_{j} T_{0}\right), \quad T_{0}=2 \pi / \Omega_{0}
$$

For convenience, we will put $\rho_{0}=\rho_{j}-\bar{\rho}_{l}$. When $j=l$ (fundamental resonance) we have $\rho_{0}=(-1)^{k}$, but when $j>l$ (combination resonance) $\bar{\rho}_{0}$ is a complex multiplier. In the second case there is also a double multiplier , but because of the symmetry of the multipliers about the real axis, it will suffice to consider the behaviour of one of them. The double multiplier $\rho_{0}$ is semi-simple, since it has two corresponding linearly independent eigenvectors $\mathbf{w}_{j}$ and $\overline{\mathbf{w}}_{l}$, and also two corresponding left eigenvectors $\mathrm{v}_{j}$ and $\overline{\mathrm{v}}_{l}$ of the form (1.10).

Perturbation of the parameters leads to bifurcation of the double multiplier $\rho_{0}$ corresponding to the point $p_{0}=\left(0,0, \Omega_{0}\right)$ into two simple multipliers. Considering the perturbation of parameters along a ray $\mathbf{p}=\mathbf{p}_{0}+\mathbf{e} \varepsilon(\varepsilon \geqslant 0)$ in the direction $\mathbf{e}$, we can describe the bifurcation of a semi-simple multiplier by the expression

$$
\rho=\rho_{0}(1+\mu \varepsilon+o(\varepsilon))
$$

The two values of $\mu$ are found from the quadratic equation [9]

$$
\begin{aligned}
& \left|\begin{array}{cc}
\mathbf{v}_{j}^{T} \mathbf{F}_{1} \mathbf{w}_{j}-\rho_{0} \mu & \mathbf{v}_{j}^{T} \mathbf{F}_{1} \overline{\mathbf{w}}_{l} \\
\mathbf{v}_{l}^{T} \mathbf{F}_{1} \mathbf{w}_{j} & \overline{\mathbf{v}}_{l}^{T} \mathbf{F}_{l} \overline{\mathbf{w}}_{l}-\rho_{0} \mu
\end{array}\right|=0 \\
& \mathbf{F}_{1}=\frac{d \mathbf{F}\left(\mathbf{p}_{0}+\mathbf{e \varepsilon}\right)}{d \varepsilon}=\frac{\partial \mathbf{F}}{\partial \gamma} e_{1}+\frac{\partial \mathbf{F}}{\partial \delta} e_{2}+\frac{\partial \mathbf{F}}{\partial \Omega} e_{3}
\end{aligned}
$$

where the derivatives are evaluated at $\varepsilon=0$ and $\mathbf{p}=\mathbf{p}_{0}$. Equation (3.4) may be written in the form

$$
\begin{aligned}
& \mu^{2}+\left(x_{1}+i x_{2}\right) \mu+y_{1}+i y_{2}=0 \\
& x_{1}+i x_{2}=-\left(\mathbf{v}_{j}^{T} \mathbf{F}_{1} \mathbf{w}_{j}+\overline{\mathbf{v}}_{l}^{T} \mathbf{F}_{1} \overline{\mathbf{w}}_{l}\right) / \rho_{0} \\
& y_{1}+i y_{2}=\left(\mathbf{v}_{j}^{T} \mathbf{F}_{l} \mathbf{w}_{j} \overline{\mathbf{v}}_{l}^{T} \mathbf{F}_{l} \overline{\mathbf{w}}_{l}-\mathbf{v}_{j}^{T} \mathbf{F}_{1} \overline{\mathbf{w}}_{l} \overline{\mathbf{v}}_{l}^{T} \mathbf{F}_{1} \mathbf{w}_{j}\right) / \rho_{0}^{2}
\end{aligned}
$$

In view of (3.3), the inequality $|\rho|<1$ becomes

$$
|\rho|=\left|\rho_{0}(1+\mu \varepsilon+o(\varepsilon))\right|=1+\varepsilon \operatorname{Re} \mu+o(\varepsilon)<1
$$

Consequently, the stability condition in the first approximation reduces to the inequality

$$
\operatorname{Re} \mu<0
$$

for both roots of the quadratic equation (3.5). Hence, using Routh-Hurwitz-type conditions for the second-order polynomial with complex coefficients (3.5), we have [1]

$$
x_{1}>0 \quad\left(x_{1} y_{1}+x_{2} y_{2}\right) x_{1}-y_{2}^{2}>0
$$

We now substitute $x_{1}, x_{2}, y_{1}$ and $y_{2}$ from (3.6) into inequalities (3.9). Elementary reduction using the expressions for eigenvectors (1.10), the derivatives of monodromy matrix (2.2) and relations (1.2), (1.8), (1.9), (1.12) and (3.1), yield the stability conditions (3.9) in the form

$$
\begin{aligned}
& \pi\left(\eta_{j}+\eta_{l}\right) e_{1} / \Omega_{0}>0 \\
& \pi^{4}\left[e_{1}^{2}\left(\eta_{j}+\eta_{l}\right)^{2}\left(\eta_{j} \eta_{l} e_{1}^{2}-\xi_{1} e_{2}^{2}+k^{2}\left(e_{3}+\sigma_{+} e_{2} / k\right)^{2}\right)-\right. \\
& \left.-\left(\xi_{2} e_{2}^{2}+k\left(\eta_{j}-\eta_{l}\right)\left(e_{3}+\sigma_{+} e_{2} / k\right) e_{1}\right)^{2}\right] / \Omega_{0}^{4}>0
\end{aligned}
$$

where the coefficients $\eta_{j}, \eta_{l}, \xi_{1}, \xi_{2}$ and $\sigma_{+}$are real numbers defined by the formulae 


$$
\begin{aligned}
& \eta_{j}=\mathbf{u}_{j}^{T} \mathbf{D} \mathbf{u}_{j}, \quad \xi_{1}+i \xi_{2}=\frac{c_{-k}^{(j l)} c_{k}^{(j)}}{\omega_{j} \omega_{l}}, \quad \sigma_{ \pm}=-\frac{\omega_{l} c_{0}^{(j j)} \pm \omega_{j} c_{0}^{(l l)}}{2 \omega_{j} \omega_{l}} \\
& c_{k}^{(j)}=\frac{1}{2 \pi} \int_{0}^{2 \pi} \mathbf{u}_{l}^{T} \mathbf{B}(\tau) \mathbf{u}_{j} e^{i k \tau} d \tau
\end{aligned}
$$

Note that the constants $\eta_{j}$ and $\eta_{l}$ are positive, because of the assumption that the matrix $D$ of dissipative forces is positive-definite. Expressing the vector $\mathbf{e}$ in terms of the other quantities in the relation $\mathbf{p}=\mathbf{p}_{0}+\mathbf{e} \varepsilon$, we obtain

$$
\mathbf{e}=\left(e_{1}, e_{2}, e_{3}\right)=\left(\mathbf{p}-\mathbf{p}_{0}\right) / \varepsilon=(\gamma, \delta, \Delta \Omega) / \varepsilon, \quad \Delta \Omega=\Omega-\Omega_{0}
$$

Substituting these expressions into (3.10) and cancelling out positive factors in the resulting inequalities, we obtain relations which determine the stability domain in the first approximation:

$$
\begin{aligned}
& \gamma>0, \quad \gamma^{2}\left(\eta_{j}+\eta_{l}\right)^{2}\left[\eta_{j} \eta_{l} \gamma^{2}-\xi_{1} \delta^{2}+k^{2}\left(\Delta \Omega+\sigma_{+} \delta / k\right)^{2}\right]- \\
& -\left[\xi_{2} \delta^{2}+k\left(\eta_{j}-\eta_{l}\right)\left(\Delta \Omega+\sigma_{+} \delta / k\right) \gamma\right]^{2}>0
\end{aligned}
$$

The first condition (3.13) means that dissipative forces are present. The second condition defines the form of the stability domain in the space of the three parameters $p=(\gamma, \delta, \Omega)$.

Finally, let us consider a critical frequency $\Omega_{0}$, which satisfies the condition

$$
\omega_{j}-\omega_{l}=k \Omega_{0}, \quad j>l
$$

for some natural number $k$ (a difference combination resonance). Here there is a double multiplier $\rho_{0}=\rho_{j}=\rho_{l}$ to which two linearly independent eigenvectors $w_{j}$ and $w_{l}(1.10)$ correspond. Reasoning by analogy and replacing $\omega_{l}$ by $-\omega_{l}$ in all the relations, we determine the stability domain in the neighbourhood of the point $\mathbf{p}_{0}=\left(0,0 \Omega_{0}\right)$ in the first approximation

$$
\begin{aligned}
& \gamma>0, \quad \gamma^{2}\left(\eta_{j}+\eta_{l}\right)^{2}\left[\eta_{j} \eta_{l} \gamma^{2}+\xi_{l} \delta^{2}+k^{2}\left(\Delta \Omega+\sigma_{-} \delta / k\right)^{2}\right]- \\
& -\left[\xi_{2} \delta^{2}-k\left(\eta_{j}-\eta_{l}\right)\left(\Delta \Omega+\sigma_{-} \delta / k\right) \gamma\right]^{2}>0
\end{aligned}
$$

where the real coefficients $\eta_{j}, \eta_{l}, \xi_{1}, \xi_{2}$ and $\sigma_{-}$are defined by relations (3.11).

We will analyse the geometry of the instability domains (of parametric resonance) in the following most frequently encountered cases.

1. Suppose the parametric excitation matrix $\mathbf{B}(\Omega t)$ is symmetric. Then the quantities $c_{-k}^{j l}$ and $c_{k}^{l j}$ are complex conjugates. Consequently, $\xi_{2}=0$, while the quantity $\xi_{1}$ in (3.11) has the form

$$
\begin{aligned}
& \xi_{1}=\frac{c_{-k}^{(j l)} c_{k}^{(j)}}{\omega_{j} \omega_{l}}=\frac{\left(a_{k}^{(j l)}\right)^{2}+\left(b_{k}^{(j l)}\right)^{2}}{4 \omega_{j} \omega_{l}} \geqslant 0 \\
& \left\{\begin{array}{l}
a_{k}^{(j l)} \\
b_{k}^{(j l)}
\end{array}\right\}=\frac{1}{\pi} \int_{0}^{2 \pi} \mathbf{u}_{j}^{T} \mathbf{B}(\tau) \mathbf{u}_{l}\left\{\begin{array}{l}
\cos k \tau \\
\sin k \tau
\end{array}\right\} d \tau
\end{aligned}
$$

In the case of fundamental resonance (3.1), the second stability condition (3.13) leads, after cancellation of a positive factor, to an inequality which defines the parametric resonance domain (PRD) in the first approximation:

$$
\eta_{j} \eta_{l} \gamma^{2}-\xi_{l} \delta^{2}+4 k^{2} \eta_{j} \eta_{l}\left(\eta_{j}+\eta_{l}\right)^{-2}\left(\Delta \Omega+\sigma_{+} \delta / k\right)^{2} \leqslant 0
$$

Since the quantities $\eta_{j}$ and $\eta_{l}$ are positive while $\xi_{1}$ is non-negative, it follows that, if $\xi_{1} \neq 0$, then condition (3.17) defines the interior of a cone in the three-dimensional parameter space $\mathbf{p}=(\gamma, \delta, \Omega)$ (Fig. 1). The cone axis, which is formed by the centres of sections of the cone by planes $\delta=$ const, is defined by the equations

$$
\gamma=0, \quad \Omega-\Omega_{0}+\sigma_{+} \delta / k=0
$$




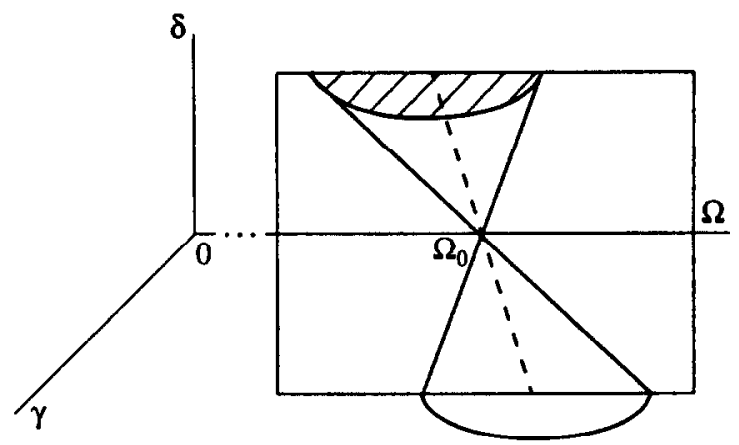

Fig. 1

In the case of parametric excitation with zero mean $c_{0}^{(j)}=c_{0}^{(l)}=0$, we have $\sigma_{+}=0$, and consequently the cone axis is parallel to the $0 \delta$ axis. The stability domain is the exterior of the cone.

If the number $k$ defining the order of the resonance (3.1) is increased, with $\omega_{l}$ and $\omega_{l}$ remaining fixed, the coefficient $\xi_{1}$ decreases as the square of the absolute value of the coefficient of the Fourier series. As a result, the instability cone narrows rapidly as $k$ increases, while the cone axis (3.18) straightens out. A section of the cone by a plane $\delta=$ const (for fixed amplitude of parametric excitation) is an ellipse which, as $k$ increases, flattens out in the direction of the $0 \Omega$ axis (Fig. 2). This is due to the presence of the factor $k^{2}$ in (3.17). Since the denominator in (3.11) contains the frequency product $\omega_{j} \omega_{l}$, it follows that as $j$ and $l$ increase, the quantities $\xi_{1}$ and $\left|\sigma_{+}\right|$typically decrease. As a result, the instability cone becomes narrower, while its axis straightens out as $j$ and $l$ are increased, that is, at resonances at higher frequencies.

If the periodic matrix function $B(\tau)$ has a finite number of terms in its Fourier expansion, the coefficient $\xi_{1}$ will vanish beginning from some $k$. This means that in the first approximation the PRD (3.17) degenerates into a straight line (3.18). To analyse the form of PRD in this case, higher-order approximations must be considered. This type of degeneration is familiar from Mathieu's equation, where the boundary of the PRD has cusps of different orders [10]. It follows from the results obtained that such degeneration occurs in three-dimensional parameter space if there are dissipative forces (there is no PRD, or the PRD forms narrow wedges with tangent rays (3.18) when $\delta>0$ and $\delta<0$ ).

In the case of difference combination resonance (3.14), stability condition (3.15) yields the PRD as an inequality:

$$
\eta_{j} \eta_{l} \gamma^{2}+\xi_{l} \delta^{2}+4 k^{2} \eta_{j} \eta_{l}\left(\eta_{j}+\eta_{l}\right)^{-2}\left(\Delta \Omega+\sigma_{-} \delta / k\right)^{2} \leqslant 0
$$

Note that inequalities (3.17) and (3.19) differ only in the sign of the second term and the coefficients $\sigma_{ \pm}$. Therefore, if $\xi_{1} \neq 0$ (the non-degenerate case), only one of these inequalities defines a cone, while the other defines a point $\gamma=\delta=\Delta \Omega=0$ (no resonance). Consequently, if $\xi_{1}$ is positive, there is no domain of difference combination resonance. Note that in Hamiltonian systems (without dissipation) the absence of difference combination resonance was known previously [1].

2. Suppose the parametric excitation matrix has the form

$$
\mathbf{B}(\Omega t)=\varphi(\Omega t) \mathbf{B}_{0}
$$

where $\mathbf{B}_{0}$ is an arbitrary matrix, independent of time, and $\varphi(t)$ is a $2 \pi$-periodic scalar function. Here the product $c_{-k}^{j l} c_{k}^{l j}$ in (3.11) is a real number. Consequently, $\xi_{2}=0$ and the coefficient $\xi_{1}$ is

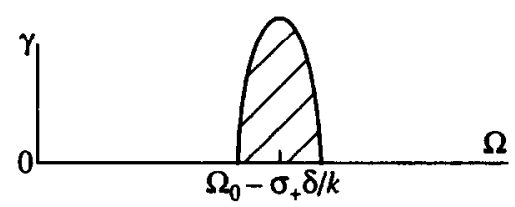

Fig. 2 


$$
\begin{gathered}
\xi_{1}=c_{j l} \frac{\alpha_{k}^{2}+\beta_{k}^{2}}{4 \omega_{j} \omega_{l}} \\
c_{j l}=\mathbf{u}_{j}^{T} \mathbf{B}_{0} \mathbf{u}_{l} \mathbf{u}_{l}^{T} \mathbf{B}_{0} \mathbf{u}_{j}, \quad\left\{\begin{array}{l}
\alpha_{k} \\
\beta_{k}
\end{array}\right\}=\frac{1}{\pi} \int_{0}^{2 \pi} \varphi(\tau)\left\{\begin{array}{l}
\cos k \tau \\
\sin k \tau
\end{array}\right\} d \tau
\end{gathered}
$$

The second stability condition (3.13) in the case of fundamental resonance and sum combination resonance (3.1) yields the PRD (3.17). In the case of difference combination resonance (3.14) the PRD has the form of (3.19). In the non-degenerate case $\xi_{1} \neq 0$, the sign of $\xi_{1}$ is like that of $c_{j l}$. In the case of fundamental resonance $c_{i j} \geqslant 0$, so that, provided that $c_{i j} \neq 0$, a fundamental resonance domain exists, described by the cone (3.17). As to the existence of combination resonance domains, this depends on the sign of $c_{j l}$. If $c_{j l}>0$, only a domain of sum combination resonance exists, while if $c_{j l}<0$, only a domain of difference combination resonance exists. The form of the PRD (a cone) depends on the order $\mathrm{k}$ of the resonance and the frequencies $\omega_{j}$ and $\omega_{l}$, as in the previously described case $1^{\circ}$. If $c_{j l}=0$, the resonance domain either does not exist or is degenerate (the first approximation yields a straight line).

Our results may by formulated as follows.

Theorem 1. If the exitation matrix is symmetric, $\mathbf{B}(\tau)=\mathbf{B}^{T}(\tau)$, then system (1.1) is subject only to fundamental resonances (1.13) and sum combination resonances of type (1.14). In the case of the matrix $\mathbf{B}(\tau)=\varphi(\tau) \mathbf{B}_{0}$, where $\varphi(\tau)$ is a periodic scalar function and $\mathbf{B}_{0}$ is a time-independent matrix, one obtains fundamental resonances (1.13) and, depending on the sign of the constant $c_{l j}$ in (3.21), either sum combination resonances $\left(c_{j l}>0\right)$ or difference combination resonances $\left(c_{j l}<0\right)$. In the three-dimensional space of the parameters $\gamma, \delta$ and $\Omega$, the domains of fundamental and sum combination resonance are described by the cones (3.17), and the domains of difference combination resonance are described by the cones (3.19).

The cases 1 and 2 just considered correspond to the most widespread forms of parametric excitation. In other cases, on can use the stability conditions (3.13) and (3.15) to construct three-dimensional PRDs.

\section{THE INFLUENCE OF DISSIPATIVE FORCES ON THE PARAMETRIC RESONANCE DOMAIN}

Let us fix a value of the parameter $\gamma>0$ and consider the case in which the condition $\xi_{2}=0$ is satisfied, say case 1 or 2 of Section 3. Then the PRDs in the first approximation have the form of (3.17) and (3.19). Depending on the sign of $\xi_{1}$, the PRD is either non-existent or contained in the interior of hyperbolas (sections of the cone by a plane $\gamma=$ const) in the plane of the two parameters $\delta$ and $\Omega$ (Fig. 3). The asymptotes of the hyperbolas are defined by the equations

$$
\left|\xi_{1}\right|^{1 / 2} \delta \pm 2 k\left(\eta_{j} \eta_{l}\right)^{1 / 2}\left(\eta_{j}+\eta_{l}\right)^{-1}\left(\Delta \Omega+\sigma_{s} \delta / k\right)=0
$$

where the subscript $s$ denotes + for resonances (3.1) and - for resonances (3.14).

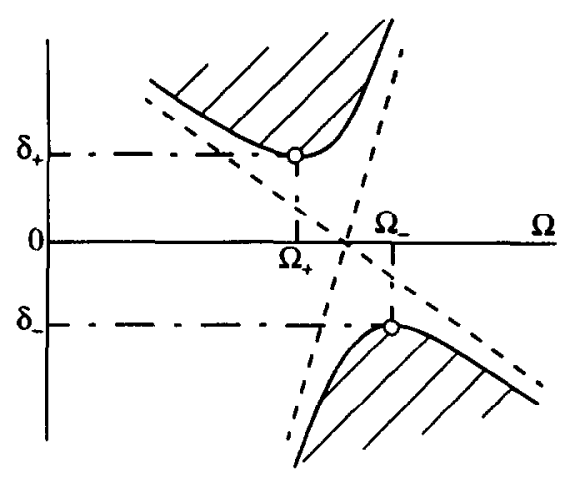

Fig. 3 
Note that $\sigma_{+}=\sigma_{-}=0$ in the case when the mean value of the matrix $\mathbf{B}(\Omega t)$ over a period is zero. Increasing the parameter $\gamma$, which defines the magnitude of the dissipative forces, leads to a decrease in the PRD in the $\delta, \Omega$ plane.

Using expressions (3.17) or (3.19), depending on the type of resonance, we find in the first approximation the minimum (critical) amplitude of excitation $|\delta|$ at which parametric resonance can occur

$$
\delta_{ \pm}= \pm\left|\eta_{j} \eta_{l} / \xi_{1}\right|^{1 / 2} \gamma
$$

The quantities $\delta_{ \pm}$correspond to excitation frequencies

$$
\Omega_{ \pm}=\Omega_{0}-\sigma_{s} \delta_{ \pm} / k
$$

where $\Omega_{0}$ is the resonance frequency (3.1) or (3.14) (Fig. 3).

Theorem 2. At small fixed values of the dissipation parameter $\gamma>0$, when $\xi_{2}=0$, the boundary of the parametric resonance domain is described in the first approximation by a hyperbola (3.17), corresponding to fundamental and sum combination resonances $\left(\xi_{1}>0\right)$, or a hyperbola (3.19), corresponding to difference combination resonance $\left(\xi_{1}<0\right)$. The minimum (critical) values of the excitation amplitude and corresponding frequencies are defined by (4.2) and (4.3).

Using relation (3.17) and (3.19), let ut investigate the limit of the PRD as $\gamma \rightarrow+0$, that is, in the case of infinitesimal dissipation. In the first approximation, for the case of fundamental resonance (3.1) $(j=l)$, the domain has the form

$$
\xi_{1} \delta^{2}>k^{2}\left(\Delta \Omega+\sigma_{+} \delta / k\right)^{2}
$$

In the case of sum combination resonance $(3.1)(s=+)$ or difference combination resonance (3.14) $(s=-)$, the domain is defined by the relation

$$
s \xi_{l} \delta^{2}>4 k^{2} \eta_{j} \eta_{l}\left(\eta_{j}+\eta_{l}\right)^{-2}\left(\Delta \Omega+\sigma_{s} \delta / k\right)^{2}
$$

The combination resonance domain in the limit, as $\gamma \rightarrow+0$ obviously depends on the ratio $\eta_{j} / \eta_{l}$ of the magnitudes of the dissipative forces associated with the $j$-th and $l$-th natural modes of the unperturbed system. If $\eta_{j}=\eta_{l}$, the combination resonance domain is minimal, while if $\eta_{j} \ll \eta_{l}$ or $\eta_{j} \gg \eta_{l}$ it is maximal and consists of two vertical angles whose magnitudes tend to $\pi$ as $\eta_{j} / \eta_{l} \rightarrow 0$ or $\eta_{j} / \eta_{l} \rightarrow \infty$ (Fig. 4).

Now assume that $\gamma=0$ and consider the case $\mathbf{B}(\tau)=\mathbf{B}^{T}(\tau)$ or $\mathbf{B}\left(\tau_{0}+\tau\right)=\mathbf{B}\left(\tau_{0}-\tau\right)$, when the characteristic polynomial of the monodromy matrix is reciprocal [1]. In that case the system is stable (not asymptotically) if and only if all the multipliers are semi-simple (the number of eigenvectors is equal to the algebraic multiplicity of the multiplier) and lie on the unit circle. Using relations (3.3) and (3.5), we find the stability condition for resonances (3.1) and (3.14) to be

$$
\operatorname{Re} \mu=0 \Leftrightarrow x_{1}=0, \quad y_{2}=0, \quad x_{2}^{2}+4 y_{1}>0
$$

Substituting the values of $x_{1}, x_{2}, y_{1}$ and $y_{2}$ from (3.6) and using relations (1.2), (1.8)-(1.12) and (2.2), as well as the assumption $\xi_{2}=0$, we find the PRD in the first approximation:

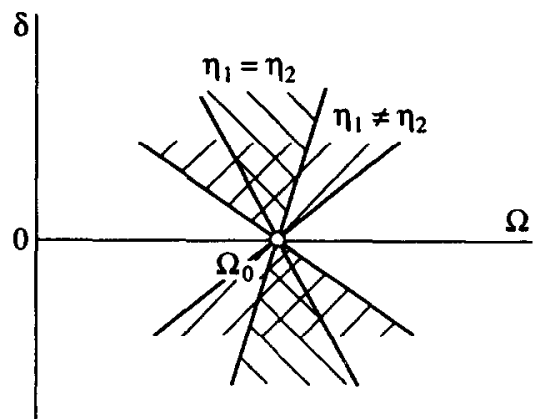

Fig. 4 


$$
s \xi_{1} \delta^{2} \geqslant k^{2}\left(\Delta \Omega+\sigma_{s} \delta / k\right)^{2}
$$

where $s=+$ for resonances (3.1) and $s=-$ for resonances (3.14).

It can be scen by comparing incqualitics (4.4) and (4.7) that the stability domains for a system with infinitesimal dissipation $(\gamma \rightarrow+0)$ and a system without dissipation $(\gamma=0)$ coincide in the case of fundamental resonances (3.1) $(j=l)$. In the case of combination resonance, however, the domains (4.5) and (4.7) coincide only when $\eta_{j}=\eta_{l}$, being different if $\eta_{j} \neq \eta_{l}$, and moreover the combination resonance domain of a system with dissipation is always larger. If the mean value of the periodic matrix $\mathbf{B}(\tau)$ vanishes, we have $\sigma_{+}=0$ and $\sigma_{-}=0$. In these cases formulae (4.4), (4.5) and (4.7) are the same as those obtained previously [1]. An increase in the combination resonance domain when infinitesimal dissipation is introduced has been observed in various mechanical systems $[1,3,11]$. This effect is analogous to the well-known paradox of the destabilization of a non-conservative system by small dissipative forces in the case of autonomous systems [12].

\section{A SYSTEM WITH ONE DEGREE OF FREEDOM}

In the case of one degree of freedom $(m=1)$, system (1.1) may be written as Hill's equation with damping

$$
\ddot{y}+\gamma \dot{y}+\omega^{2}(1+\delta \varphi(\Omega t)) y=0
$$

The presence of just one natural frequency $\omega$ means that there is no combination resonance. There is fundamental resonances when $\Omega_{0}=2 \omega / k(k=1,2, \ldots)$, and the corresponding three-dimensional fundamental resonance domains (3.17) have the form

$$
\gamma^{2}-\frac{\alpha_{k}^{2}+\beta_{k}^{2}}{4} \omega^{2} \delta^{2}+k^{2}\left(\Delta \Omega-\frac{\alpha_{0} \omega}{2 k} \delta\right)^{2} \leqslant 0
$$

where $\alpha_{k}$ and $\beta_{k}$ are the Fourier coefficients of the function $\varphi(\tau)$ of (3.21). For fixed small values of $\gamma>0$, the critical amplitudes and frequencies of excitation are, by formulae (4.2) and (4.3),

$$
\delta_{ \pm}= \pm \frac{2 \gamma}{\omega\left(\alpha_{k}^{2}+\beta_{k}^{2}\right)^{1 / 2}}, \quad \Omega_{ \pm}=\Omega_{0}+\frac{\alpha_{0} \omega}{2 k} \delta_{ \pm}
$$

In the case when $\varphi(\tau)=\cos \tau$ (Mathieu's equation with damping) and $k=1$, formulae (5.2) and (5.3) are identical, after some reduction with the approximate formulae for the first fundamental resonances domain and critical amplitude presented in [3].

\section{EXAMPLES}

Let us consider Bolotin's problem [3,13] of the dynamic stability of the two-dimensional bending mode of an elastic beam (Fig. 5). It is assumed that the beam, of length $l$, is freely supported at its ends and loaded in the plane of maximum stiffness by periodic torques $M(\Omega t)=\delta \varphi(\Omega t)$, where $\varphi(\tau)$ is a $2 \pi$-periodic function. The flexural-torsional vibrations that ensue from that plane are described by the following equations [13]

$$
\begin{aligned}
& m \ddot{w}+\gamma m d_{1} \dot{w}+E J w^{\prime \prime \prime \prime}+\delta \varphi(\Omega t) \theta^{\prime \prime}=0 \\
& m r^{2} \ddot{\theta}+\gamma m r^{2} d_{2} \dot{\theta}+\delta \varphi(\Omega t) w^{\prime \prime}-G I \theta^{\prime \prime}=0
\end{aligned}
$$

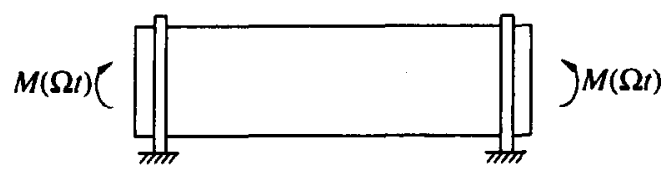

Fig. 5 
where $w(x, t)$ is the transverse deflection of the beam, $\theta(x, t)$ is the angle of rotation of the cross-section, $E J$ and $G I$ are the bending and torsion stiffnesses of the beam, respectively, $m$ is the mass per unit length of the beam, $r$ is its cross-sectional radius of inertia, $\gamma$ is the parameter of dissipative forces (viscous friction), $d_{1}$ and $d_{2}$ are fixed constants defining the magnitude of the friction forces associated with bending and torsion; dots denote differentiation with respect to time $t$, and primes denote differentiation with respect to the coordinate $x$. The boundary conditions are

$$
x=0, l: \quad w=w^{\prime \prime}=\theta=0
$$

A solution of system (6.1), (6.2) is sought in series form [3]

$$
w(x, t)=\sum_{n=1}^{\infty} W_{n}(t) \sin \frac{n \pi x}{l}, \quad \theta(x, t)=\sum_{n=1}^{\infty} \Theta_{n}(t) \sin \frac{n \pi x}{l}
$$

where $W_{n}(t)$ and $\Theta_{n}(t)$ are unknown functions of time. Substituting these series into Eqs (6.1), we obtain a system of ordinary differential equations for $W_{n}(t)$ and $\Theta_{n}(t)$ of the form (1.1) with

$$
\begin{aligned}
& \mathbf{M}=\operatorname{diag}\{1,1\}, \quad \mathbf{D}=\operatorname{diag}\left\{d_{1}, d_{2}\right\}, \quad \mathbf{C}=\operatorname{diag}\left\{\omega_{n 1}^{2}, \omega_{n 2}^{2}\right\} \\
& \mathbf{B}(\Omega t)=\varphi(\Omega t)\left\|\begin{array}{cc}
0 & -\chi^{2} / m \\
-\chi^{2} /\left(r^{2} m\right) & 0
\end{array}\right\|, \quad \mathbf{y}=\left\|\begin{array}{c}
W_{n} \\
\Theta_{n}
\end{array}\right\| \\
& \omega_{n 1}=\chi^{2} \sqrt{\frac{E J}{m}}, \quad \omega_{n 2}=\frac{\chi}{r} \sqrt{\frac{G l}{m}}, \quad n=1,2, \ldots ; \chi=\pi \frac{n}{l}
\end{aligned}
$$

where $\omega_{n 1}$ and $\omega_{n 2}$ are the natural frequencies of flexural and torsional vibrations of the beam, respectively, whose eigenvectors are

$$
u_{n 1}=\left\|\begin{array}{l}
1 \\
0
\end{array}\right\|, \quad u_{n 2}=\left\|\begin{array}{l}
0 \\
1
\end{array}\right\|
$$

Let us investigate the stability of system (1.1), (6.4) for some fixed value of $n$. Since $\mathbf{B}(\Omega t)=\varphi(\Omega t) \mathbf{B}_{0}$, where $\mathbf{B}_{0}$ is fixed matrix, the system belongs to the type considered in Subsection 2 of Section 3 . The quantities $c_{j l}$, computed from formula (3.21), are

$$
c_{11}=c_{22}=0, \quad c_{12}=\frac{\pi^{4} n^{4}}{l^{4} r^{2} m^{2}}>0
$$

Consequently, there are no domains of difference combination resonance, and the domains of fundamental resonance are degenerate (their analysis requires the computation of higher-order approximations). By inequality (3.17), the domains of sum combination resonances at frequencies close to $\Omega_{0}=\left(\omega_{n 1}+\omega_{n 2}\right) / k(k=1,2, \ldots)$ have the form

$$
d_{1} d_{2} \gamma^{2}-\frac{c_{12}\left(\alpha_{k}^{2}+\beta_{k}^{2}\right)}{4 \omega_{n 1} \omega_{n 2}} \delta^{2}+4 k^{2} \frac{d_{1} d_{2}}{\left(d_{1}+d_{2}\right)^{2}} \Delta \Omega^{2} \leqslant 0
$$

where $\alpha_{k}$ and $\beta_{k}$ are defined in (3.21).

Numerical computations were carried out for the case

$$
\begin{array}{ll}
n=l, \quad \varphi(\tau)=\cos \tau, & d_{1}=d_{2}=1, \quad \omega_{n 1}=1 c^{-1}, \quad \omega_{n 2}=\sqrt{5} c^{-1} \\
l^{2} m=\pi^{2} / 4 \mathrm{~kg} / \mathrm{cm}, & r^{2}=4 / \sqrt{5} \mathrm{~cm}^{2}
\end{array}
$$

Figure 6 (the continuous curves) shows the boundary of the first combination resonance domain $(k=1)$ in the first approximation (6.5). The dashed curves show the boundary of the combination resonance domain obtained by numerical computation of the monodromy matrix at different values of the parameters $\gamma, \delta$ and $\Omega$. Equations (1.3) were integrated using the Runge-Kutta method. Figure 6 shows that there is good agreement between the exact (numerically determined) and approximate combination resonance domains, up to values of the amplitude $\delta \approx 0.8$. 


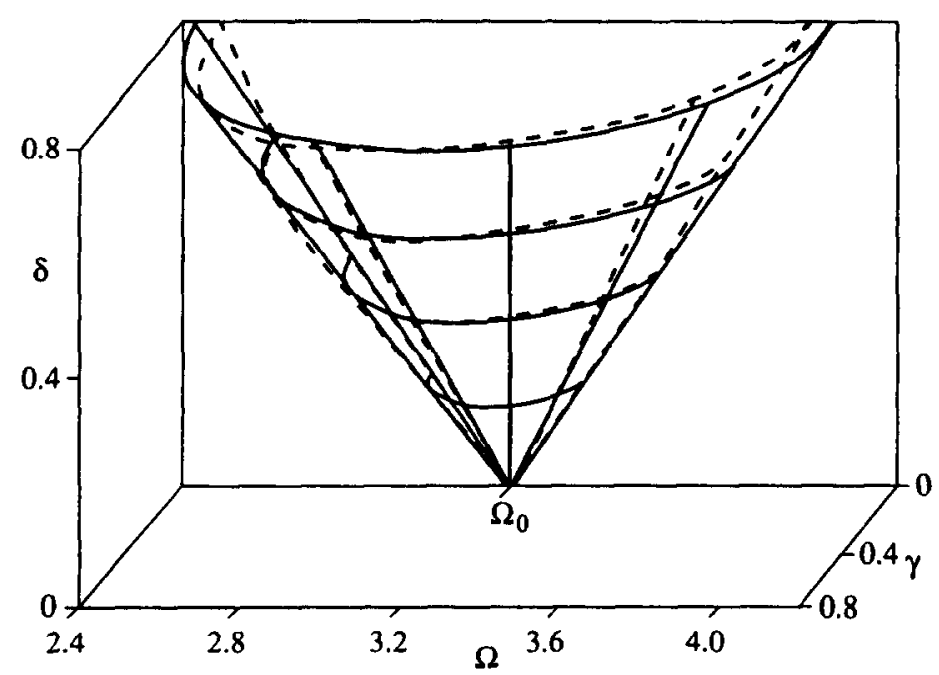

Fig. 6

2. As a second example, consider an elastic rod of length $l$ of variable cross-section, loaded by a longitudinal periodic force $P=P_{0}+\delta \varphi(\Omega t)$ where $\delta$ and $\Omega$ are the amplitude and frequency of excitation and $P_{0}$ is a fixed value, less than the critical Euler force $P_{\mathrm{E}}$. The equation of small vibrations of the rod has the form [3]

$$
m(x) \ddot{w}+\gamma s(x) \dot{w}+\left[P_{0}+\delta \varphi(\Omega t)\right] w^{\prime \prime}+\left(E J(x) w^{\prime \prime}\right)^{\prime \prime}=0
$$

where $w(x, t)$ is the deflection of the rod, $E J(x)$ is its stiffness, $m(x)$ is the mass per unit length, $s(x)$ is the thickness of the rod and $\gamma$ is the coefficient of viscous friction. The rod is elastically clamped at both ends with stiffnesses $c_{1} \geqslant 0$ and $c_{2} \geqslant 0$.

Then the boundary conditions are

$$
w(0)=w(l)=0 \quad\left(-c_{1} w^{\prime}+E J w^{\prime \prime}\right)_{x=0}=\left(c_{2} w^{\prime}+E J w^{\prime \prime}\right)_{x=1}=0
$$

The limiting cases $c_{1}=c_{2}=0$ and $c_{1}^{-1}=c_{2}^{-1}=0$ correspond to hinged and clamped ends, respectively. A solution of system (6.6), (6.7) will be sought in series form

$$
w(x, t)=\sum_{n=1}^{\infty} W_{n}(t) u_{n}(x)
$$

where $W_{n}(t)$ are unknown functions of time and $u_{n}(x)$ are the natural modes of vibration of a beam compressed by a constant force $P_{0}$; the latter functions are determined from the eigenvalue problem $\left(\omega_{n}\right.$ are the vibration frequencies)

$$
\begin{gathered}
\left(E J(x) u_{n}^{\prime \prime}\right)^{\prime \prime}+P_{0} u_{n}^{\prime \prime}-\omega_{n}^{2} m(x) u_{n}=0 \\
u_{n}(0)=u_{n}(l)=0 \quad\left(-c_{1} u_{n}^{\prime}+E J u_{n}^{\prime \prime}\right)_{x=0}=\left(c_{2} u_{n}^{\prime}+E J u_{n}^{\prime \prime}\right)_{x=l}=0
\end{gathered}
$$

The eigenfunctions satisfy the orthonormality conditions

$$
\int_{0}^{l} m u_{i} u_{j} d x=\delta_{i j}
$$

where $\delta_{i j}$ is the Kronecker delta

Substituting series (6.8) into Eq. (6.6) and using Eq. (6.9), we obtain the equation

$$
\sum_{n=1}^{\infty}\left[m \ddot{W}_{n} u_{n}+\gamma s \dot{W}_{n} u_{n}+\delta \varphi(\Omega t) W_{n} u_{n}^{\prime \prime}+\omega_{n}^{2} m W_{n} u_{n}\right]=0
$$


from which, multiplying by $u_{j}(x)(j=1,2, \ldots)$, integrating with respect to $x$ from 0 to $l$ and using integration by parts and conditions (6.10) and (6.11), we obtain a system of equations (1.1) in which the matrices $\mathbf{M}$ and $\mathbf{C}$ are diagonal, while $\mathbf{D}$ and $\mathbf{B}(\Omega t)$ are symmetric:

$$
\begin{aligned}
& \mathbf{M}=\left\|\delta_{i j}\right\|, \quad \mathbf{C}=\left\|\omega_{i}^{2} \delta_{i j}\right\|, \quad \mathbf{D}=\left\|d_{i j}\right\|, \quad \mathbf{B}=\varphi(\Omega t)\left\|b_{i j}\right\| \\
& d_{i j}=\int_{0}^{l} s u_{i} u_{j} d x, \quad b_{i j}=-\int_{0}^{l} u_{i}^{\prime} u_{j}^{\prime} d x \quad\left(d_{j j}>0, \quad b_{j j}<0\right)
\end{aligned}
$$

We will investigate the stability of the system at small $\delta$ and $\gamma$. By Theorem 1 , only fundamental and sum combination resonances are possible. All the components of the eigenvector $\mathbf{u}_{i}$ belonging to frequency $\omega_{i}$ are zero, except for a one in the $i$-th position. The first approximation equation for the three-dimensional fundamental resonance domain $\left(\Omega_{0}=2 \omega_{j} / k\right)$ and combination resonance domain $\left(\Omega_{0}=\left(\omega_{j}+\omega_{l}\right) / k, j>l\right)(3.17)$ have the following form, taking relations (3.21) and (6.12) into account

$$
d_{j j} d_{l l} \gamma^{2}-\frac{b_{j l}^{2}\left(\alpha_{k}^{2}+\beta_{k}^{2}\right)}{4 \omega_{j} \omega_{l}} \delta^{2}+4 k^{2} \frac{d_{j j} d_{l l}}{\left(d_{j j}+d_{l l}\right)^{2}}\left(\Delta \Omega-\frac{\alpha_{0}\left(\omega_{j} b_{l l}+\omega_{l} b_{j j}\right)}{4 k \omega_{j} \omega_{l}} \delta\right)^{2} \leqslant 0
$$

The quantities $\alpha_{k}$ and $\beta_{k}$ are defined in (3.21). The critical values of the amplitude (4.2) and frequency (4.3) for fixed small $\gamma>0$ are

$$
\delta_{ \pm}= \pm\left(\frac{4 \omega_{j} \omega_{l} d_{j j} d_{l l}}{b_{j l}^{2}\left(\alpha_{k}^{2}+\beta_{k}^{2}\right)}\right)^{1 / 2} \gamma, \quad \Omega_{ \pm}=\Omega_{0}+\frac{\alpha_{0}\left(\omega_{j} b_{l l}+\omega_{l} b_{j j}\right)}{4 k \omega_{j} \omega_{l}} \delta_{ \pm}
$$

In the case of a uniform rod, clamped or hinged at its ends, with $k=1$ and a longitudinal load $P=\delta \cos / \Omega t$, formulae (6.13) and (6.14) are identical with those presented in [11].

\section{REFERENCES}

1. YAKUBOVICH, V. A., and STARZHINSKII, V. M., Parametric Resonance in Linear Systems. Nauka, Moscow, 1987.

2. HSU, C. S., On the parametric excitation of a dynamic system having multiple degrees of freedom. Trans. ASME, Ser. E, J. Appl. Mech., 1963, 30, 3, 367-372.

3. V. V. BOLOTIN (ed.), Vibrations in Engineering, Vol. 1. Vibrations of Linear System. Mashinostroyeniye, Moscow, 1999.

4. SCHMIDT, G., Parameterregte Schwingungen. Akademie Verlag, Berlin, 1975.

5. CHELOMEI, S. V., The dynamic stability of elastic system. Dokl. Akad. Nauk SSSR, 1980, 252, 2, 307-310.

6. SEYRANIAN, A. P., SOLEM, F. and PEDERSEN, P., Stability analysis for multiparameter linear periodic systems. Archive Appl. Mech., 1999, 69, 3, 160-180.

7. MAILYBAYEV, A. A. and SEYRANIAN A. P., The boundaries of the parametric resonance domain. Prikl. Mat. Mekh., $2000,64,6,947-962$.

8. SEYRANIAN, A. P., Resonance domains for Hill's equation with damping. Dokl. Ross. Akad. Nauk, 2001, 376, 1, $44-47$.

9. SEYRANIAN, A. P., Sensitivity analysis of eigenvalues and development of instability. Strojnicky Casopis, 1991, 42, 3, $193-208$.

10. ARNOLD, V. I., Remarks on perturbation theory for Mathieu-type problems. Uspekhi Mat. Nauk, 1983, 38, 4, $189-203$.

11. IWATSUBO, T., SUGIYAMA, Y. and OGINO, S., Simple and combination resonances of columns under periodic axial loads. J. Sound Vibration, 1974, 33, 2, 211-221.

12. SEYRANIAN, A. P., The stabilization of non-conservative systems by dissipative forces and indeterminacy of the critical load. Dokl. Ross. Akad. Nauk, 1996, 348, 3, 323-326

13. BOLOTIN, V. V., Dynamic stability of structures. In Nonlinear Stability of Structures. Theory and Computational Techniques, (Edited by A. N. Kounadis and W. B. Kratzig). Springer, Wien, 1995, 3-72. 